\title{
Hierarchical and Adaptive Spectrum Sensing in Cognitive Radio based Multi-hop Cellular Networks
}

\author{
Hongcheng Zhuang, Zezhou Luo, Jietao Zhang \\ Communication Technologies Lab \\ Huawei Technologies Co., Ltd. \\ Shenzhen, China \\ \{zhc, luozezhou,jtzhang\}@huawei.com
}

\author{
Halim Yanikomeroglu \\ Department of Systems and Computer Engineering \\ Carleton University \\ Ottawa, Canada \\ halim@sce.carleton.ca
}

\begin{abstract}
In a spectrum overlay system, the secondary users (SUs) with cognitive radio capability need to detect the presence of primary users promptly and reliably in order to prevent excessive interference. Likewise, to make full use of the available spectra, such systems have to attain low false alarm probability. Having a high detection probability while maintaining a low false alarm probability is challenging as these are conflicting goals; therefore, an appropriate tradeoff needs to be determined. In this paper we propose a "Hierarchical and Adaptive Spectrum Sensing (HASS)" solution to multi-hop cellular networks, which is based on cooperative sensing and soften hard detection fusion mechanisms with one-bit overhead. SUs that are able to make local detection decisions either directly report their one-bit decisions to the cognitive radio base station or they relay their decisions to other favorable SUs based on the states of their reporting channels. If SUs can not make detection decisions, they relay the observed signals to other favorable SUs for further processing. Simulation results show HASS solution improves spectrum sensing performance in multi-hop cellular networks.
\end{abstract}

Keywords- Multi-hop Cellular Network; Cognitive Radio; Cooperative Spectrum Sensing; Soften Hard Combination

\section{INTRODUCTION}

In a multi-hop cellular network (MCN) [1], a mobile station can access the cellular base station (BS) via one or more relay stations (RSs). In such networks, the cell capacity or coverage can be substantially enhanced, due to the significant reduction in link path losses via multi-hop transmissions. In addition, spectrum overlay could be achieved in a MCN based on the spatial isolation of the links (even within the same cell). The enabling technology for such flexible and efficient spectrum utilization is the cognitive radio (CR) technology [2].

Based on CR, a secondary system that has not licensed spectrum can share spectrum with a primary system owning licensed spectrum through opportunistic spectrum access [3], or share open spectrum with other secondary systems according to certain spectrum etiquette [4]. In recent years, a high number of research results have been reported in the spectrum sharing area. Especially, the results on effective spectrum sensing technologies have been getting increasing attention; for example, transmitter-based spectrum sensing technologies including energy detection [5]-[7], matched filter detection [8], and cyclostationary feature detection [9]. The matched filter is optimal but it needs the prior knowledge of the primary system. Cyclostationary feature detection can detect the signals with very low signal-to-noise ratio (SNR) but still requires some prior knowledge of the primary users (PUs). Energy detection has been widely applied since it does not require any prior knowledge of the primary signals and has much lower complexity in comparison to the other two schemes.

In practical applications of spectrum sensing, we have to take at least two aspects into consideration: i) how to design detection policy so as to obtain the accurate spectrum information in a noisy environment; ii) how to share or report detection results reliably and with low overhead. In general, detection policies include transmitter-based detection, receiverbased detection [10], and cooperative detection [11]-[13] where information from different SUs is combined (fused) to make a decision on the presence or absence of primary users. In [11] and [12], only the conventional hard combination was considered; in this case, each SU feedbacks a one-bit message regarding whether its detected energy is above a certain threshold or not. A soft combination scheme was proposed in [13], where SUs report detection results with two-bit overheads. The reliability of exchanging detection results was further discussed in [14]-[15].

Cluster-based cooperative detection mechanisms were proposed in [16]-[18]. In [16], the SU with the highest SNR in a cluster is selected to be the cluster-head. Only the clusterhead detects spectrum and then reports detection results to a base station where the final decision is made. Similar to [13], the soft detection fusion mechanism was used in [16] to enhance the reliability of detection. It was assumed that the clustering is already pre-allocated before conducting sensing. The same assumption was adopted in [17]-[18]. In [17], cluster-heads exchange local decisions with each other to obtain the sensing result of the entire network. The SU in a cluster with the highest instantaneous reporting channel gain was selected to be the cluster-head in [18]. The cluster-head receives local observations from other SUs in the same cluster and then makes a cluster decision based on the conventional hard detection fusion mechanism that divides the energy range into two regions by one energy threshold. A common receiver makes a final decision according to the cluster decisions reported from all cluster-heads.

It can be seen from above that these existing cooperative spectrum sensing mechanisms focus on only one aspect, either spectrum detecting or result reporting. In a practical CR 
system, both aspects are indispensable. Moreover, these mechanisms are impractical since the soft detection fusion mechanism with two-bit overhead increases the probability of erroneous reporting due to channel fading, while the hard detection fusion mechanism with two energy regions degrades detection performance due to influences from primary users.

In this paper, we propose a hierarchical and adaptive cooperative spectrum detecting and reporting solution to MCN to improve the entire sensing performance, benefited from diversity gains resulting from adaptive cooperation between SUs. SUs which fail to detect the absence or presence of primary users forward their observed signals to the most favorable SU for further detection; SUs which succeed in detecting but with poor reporting channel gain also forward their detection results to the most favorable SU for further processing. By employing such a hierarchical and adaptive solution, the detecting and reporting performance can be improved. Moreover, we propose a detecting and reporting RS selection algorithm, and discuss data fusion in the relay SUs.

The rest of this paper is organized as follows. Hierarchical and adaptive spectrum sensing solution to $\mathrm{MCN}$ is proposed in Section II. Simulation results are shown and discussed in Section III. Finally, we draw our conclusions in Section IV.

\section{HIERARCHICAL \& ADAPTIVE SPECTRUM SENSING (HASS) SOLUTION}

\section{A. System Model}

In HASS, we employ the energy detection mechanism for detecting the presence or absence of PUs and assume that there exists a common control channel for exchanging spectrum sensing signals. HASS divides the observed signal energy range $(Y)$ into three energy regions by two detection parameters $\lambda_{1}$ and $\lambda_{2}$, denoted by

$$
\mathrm{Y}=\left(E_{\mathrm{s}}+E_{n}\right) \begin{gathered}
H_{l} \\
>\lambda_{2} \\
<\lambda_{1}, \\
H_{0}
\end{gathered},
$$

where $E_{s}$ and $E_{n}$ are the PU signal energy and noise signal energy, respectively. The values $\lambda_{1}$ and $\lambda_{2}$ are determined from detection probability, $P_{d}$, and false alarm probability, $P_{f}$, as [19]

$$
\begin{aligned}
& \lambda_{1} \approx \log \frac{1-P_{d}}{1-p_{f}}, \\
& \lambda_{2} \approx \log \frac{P_{d}}{p_{f}} .
\end{aligned}
$$

Figure 1 shows the HASS architecture, in which a SU that is not able to detect the presence or absence of PUs, or is not able to directly report its local detection results to CR-BS, has to select a relay $\mathrm{SU}$ denoted as the cooperative sensing relay station (CS-RS).

HASS divides a sensing frame into three sub-slots. In subslot1, every SU conducts spectrum detection; in sub-slot2, a
SU may be continue to detect or report detection results to CRBS or it relays its observed signal to a favorable CS-RS; in subslot3, CS-RSs combine detection results from cooperative sensing SU (CS-SUs) with local detection result and report to CR-BS.
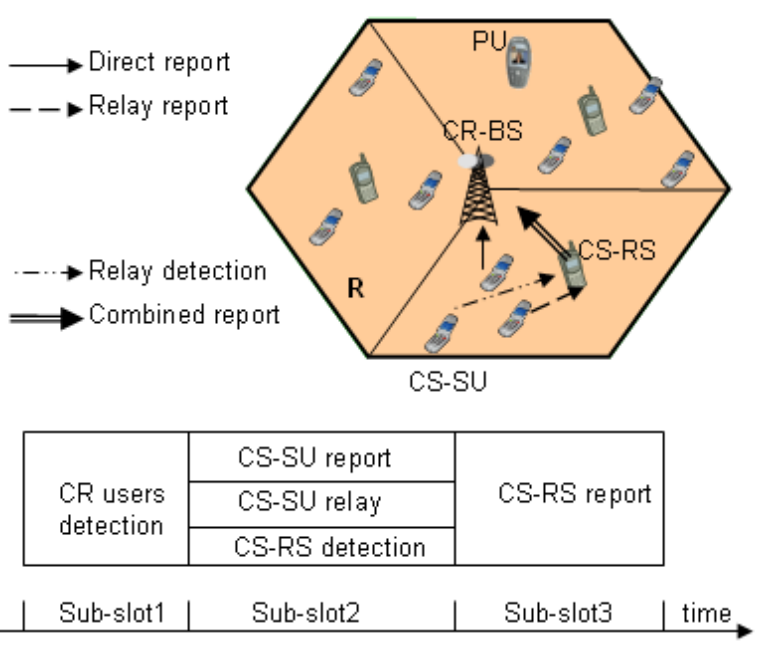

Figure 1. The HASS architecture.

In a CR cell, every SU has different spectrum detection performance due to the random activeness and location of PUs. Moreover, each has different reporting performance due to the variable reporting channel to CR-BS. Therefore, SUs should take corresponding actions based on the states of the reporting channel, relay channel, and detection channel, as shown in Table 1 .

TABLE 1. SENSING ACTIONS OF SUS

\begin{tabular}{|l|l|l|l|}
\hline $\begin{array}{l}\text { Reporting } \\
\text { Channel }\end{array}$ & $\begin{array}{l}\text { Detection } \\
\text { Channel }\end{array}$ & $\begin{array}{l}\text { Relay } \\
\text { Channel }\end{array}$ & SU Action \\
\hline 0 & 0 & 0 & Discard \\
\hline 0 & 0 & 1 & Relay detection \\
\hline 0 & 1 & 0 & Discard \\
\hline 0 & 1 & 1 & Relay report \\
\hline 1 & 0 & 0 & Discard \\
\hline 1 & 0 & 1 & Continuous detection, \\
& 1 & 0 & \begin{tabular}{l} 
Conbined report report combined report \\
\hline 1
\end{tabular} \\
\hline 1 & 1 & 1 &
\end{tabular}

Reporting channel is the channel between CR-BS and a SU. "State $=1$ " indicates that the reporting channel is reliable.

Detection channel is the channel between a SU and PUs. "State $=1$ " indicates that the SU is able to detect the presence or absence of PUs.

Relay channel is the channel between a SU and another SU. "State $=1$ " indicates that the SU can find a favorable CS-RS. 
Direct report: If the observed signal energy $Y>\lambda_{2}$ (indicating the presence of PUs), or if $Y<\lambda_{1}$ (indicating the absence of PUs), CS-SUs directly report the local detection decisions to CR-BS when the reporting channel to CR-BS is reliable.

Relay report: If a CS-SU is able to detect the presence or absence of PUs and if the reporting channel to CR-BS is not reliable, the CS-SU reports the local detection decision to a favorable CS-RS.

Relay detection: If the observed signal energy is in the range $\lambda_{1} \leq Y \leq \lambda_{2}$ (indicating an ambiguous detection result), then the CS-SU relays the observed signal to a favorable CS-RS for further spectrum sensing.

Combined report: CS-RSs do local spectrum detection and make data fusion based on their own detection results and the results reported from CS-SUs, and then report the outcome to CR-BS.

Finally CR-BS makes data fusion and broadcasts the final decision.

\section{B. Adaptive Selection of CS-RS}

In HASS, it is important to select the favorable CS-RSs, which depends on channel states. The CS-RS selection criterion in this paper is based on the equivalent channel from CS-SU to CR-BS through CS-RS. Detailed CS-RS selection procedure is described below:

1) CR-BS broadcasts the available spectrum message on the common control channel to the entire cell at the start of the sensing period by piggybacking the report reliability parameter $B E R_{\text {min }}$ (minimum bit error rate) and energy detection parameters $\lambda_{1}$ and $\lambda_{2}$.

2) SUs evaluate the channel to CR-BS based on the message and respond to CR-BS if channel qualities meet sensing report requirement.

For Rayleigh fading channel and BPSK modulation, the average report error probability is given by

$$
B E R_{i}=\int_{0}^{\infty} Q\left(\sqrt{\left.2 \gamma_{i}\right)} \frac{1}{\bar{\gamma}_{i}} \exp ^{-\frac{\gamma_{i}}{\gamma_{i}}} \mathrm{~d} \gamma_{i},\right.
$$

where $\gamma_{i}$ and $\bar{\gamma}_{i}$ are the instantaneous and average channel SNRs from the $i^{\text {th }}$ SU to CR-BS, respectively. In (3), $Q($.) is the Q-function.

SUs whose reporting channel qualities meet the requirement $\left(B E R_{i} \leq B E R_{\min }\right)$ become the candidate CS-RSs, other SUs are CS-SUs.

3) CS-SUs listen to the response of CS-RSs and evaluate the channels to CS-RS, and then build their own candidate CSRSs list.

4) If $B E R_{i}>B E R_{\min }$, CS-SUs select the most favorable CS-RS for reporting based on equivalent channel power gain of decode-and-forward (DF) [20]:

$$
\underset{j}{\arg \max }\left(G_{j, i}^{D F}=\frac{G_{j} G_{i, j}}{G_{j}-G_{i}+G_{i, j}}\right),
$$

where $G_{i, j}$ is the channel power gain from the $i$-th CS-SU to the $j$-th CS-RS, $G_{j}$ and $G_{i}$ are the channel power gains from the $j$-th CS-RS and the $i$-th CS-SU to CR-BS, respectively.

5) If $\lambda_{1} \leq Y \leq \lambda_{2}$, CS-SUs select the most favorable CS-RS for further detection based on amplify-and-forward (AF) cooperative mode:

$$
\underset{j}{\arg \max }\left(\gamma_{j, i}^{A F}=\frac{\gamma_{p u, i} \gamma_{i, j}}{\gamma_{p u, i}+\gamma_{i, j}+1}\right),
$$

where $\gamma_{p u, i}$ is the channel SNR from PUs to the $i$-th CS-SU.

The favorable CS-RS combines the signals with equal gain combining (EGC). The observed signal at CS-RS is given by

$$
y(t)=h_{p} s(t)+\sum_{\mathrm{i}=1}^{\mathrm{M}} g_{i} h_{i} h_{p i} s(t)+n(t),
$$

where $s(t)$ is the signal from PUs, $n(t)$ is the noise including forwarded noises from CS-SUs, $M$ is the number of CS-SUs relaying to the CS-RS, and $h_{p}, h_{p i}$, and $h_{i}$ are the channel gains between the CS-RS and PUs, the $i$-th CS-SU and PUs, CS-RS and the $i$-th CS-SU, respectively.

The scaling factor used by the i-th CS-SU to relay the observed signal to CS-RS, $g_{i}$, is given by

$$
g_{i}=\sqrt{\frac{P_{i}}{\left|h_{p i}\right|^{2}|s(t)|^{2}+N_{0}}},
$$

where $P_{i}$ is the transmitting power of the $i$-th CS-SU and $N_{0}$ is the noise power.

The detection SNR at the $j$-th CS-RS can be obtained by

$$
\gamma_{j}=\frac{1+{ }_{M} \times \frac{\pi}{4}}{{ }_{M}+1} \sum_{i=0}^{M} \gamma_{j, i}^{A F}
$$

where $\gamma_{j, 0}^{A F}$ is the channel SNR from PUs to the $j$-th CS-RS.

The local detection probability at CS-RS is given by

$$
P_{\text {d.local }}=P\left(Y>\lambda_{2} \mid H_{1}\right)=\int_{0}^{\infty} Q_{m(M+1)}\left(\sqrt{2 \gamma_{j}}, \sqrt{\lambda_{2}}\right) f\left(\gamma_{j}\right) \mathrm{d} \gamma_{j},
$$

where $Q(.,$.$) is the generalized Marcum Q-function, and \gamma_{j}$ follows the following distribution with the mean value $\overline{\gamma_{j}}$ [21]

$$
f\left(\gamma_{j}\right)=\frac{\gamma_{j}^{M}}{M ! \bar{\gamma}_{j}^{M+1}} \exp ^{-\frac{\bar{\gamma}_{j}}{\gamma_{j}}} .
$$




\section{Data Fusion in CS-RS and CR-BS}

Data fusion in CS-RS and CR-BS is based on the " $K$ out of $N$ " rule, as given by

$$
B= \begin{cases}H_{1}, & \text { if } \sum_{i=1}^{N} S_{i} \geq K, \\ H_{0}, & \text { otherwise }\end{cases}
$$

where $S_{i}$ denotes the reporting detection results from the twolayer spectrum sensing. If there are $K$ " 1 "s among $N$ local decisions, the decision, denoted as $B$, is " 1 ".

For CR-BS, it means that the spectrum is not available in the CR cell when $B=1$, because there are $K$ of $N$ SUs sensing the corresponding spectrum is occupied.

For a CS-RS, we classify data fusions into three types. In Type 1, a CS-RS is able to specify local detection, it sends the decision result to CR-BS and discards the reports from CS-SUs. Otherwise, the CS-RS makes relay-level decision fusion based on the detection results reported from CS-SUs according to the " $K$-out-of- $N$ " rule, and then sends it to CR-BS. This is the traditional cooperative spectrum sensing scheme.

In Type 2, a CS-RS firstly makes relay-level data fusion based on reports from CS-SUs according to the " $K$-out-of- $N$ " rule, then combines the relay-level result and the local detection result to the final result under the OR rule, and finally sends to CR-BS. The detection probability of relay-level is given by

$$
P_{d, \text { relay }}=\sum_{j=K}^{N}\left(\begin{array}{c}
N \\
j
\end{array}\right) P_{d, i}^{j}\left(1-P_{d, i}^{N-j}\right) .
$$

The detection probability of the $i^{\text {th }} \mathrm{CS}-\mathrm{SU}, P_{d . i}$, is given by

$$
P_{d . i}=P\left(Y>\lambda_{2} \mid H_{1}\right)=\int_{0}^{\infty} Q_{m}\left(\sqrt{\gamma_{i}}, \sqrt{\lambda_{2}}\right) f\left(\gamma_{i}\right) \mathrm{d} \gamma_{i},
$$

where $\gamma_{i}$ is the channel SNR from PUs to the $i^{\text {th }}$ CS-SU, following the exponential distribution with the mean value $\overline{\gamma_{i}}$

$$
f\left(\gamma_{i}\right)=\frac{1}{\bar{\gamma}_{i}} \exp ^{-\frac{\gamma_{i}}{\bar{\gamma}_{i}}} \text {. }
$$

We can get the detection probability of CS-RS by

$$
\begin{gathered}
P_{d, c s-r s}=1-\left\lfloor\left(1-P_{d, \text { relay }}\right)\left(1-B E R_{i, \text { relay }}\right)+P_{d, \text { relay }} B E R_{i, \text { relay }}\right\rfloor \times \\
{\left[\left(1-P_{d, \text { local }}\right)\left(1-B E R_{i}\right)+P_{d, \text { local }} B E R_{i}\right]}
\end{gathered}
$$

where $B E R_{i}$ and $B E R_{i, \text { relay }}$ are defined by (3), but the SNR in $B E R_{i, \text { relay }}$ is the equivalent SNR which is based on (4).

The process in Type 3 is similar to Type 2, except that CSRS sends the relay-level result and the local detection result separately to CR-BS, without combining them.

Due to the uncertainty in $N$ in our system model, the optimal value of $K$ [22] is hard to get. Instead, the ratio of $K$ to $N$ in the " $K$-out-of- $N$ " rule becomes a very important parameter in determining the sensing performance. The higher $K / N$ is, the lower the false-alarm probability. We will discuss the impact of data fusion in CS-RS and different $K / N$ in CS-RS and CR-BS in Section III.

\section{PERFORMANCE ANALYSES}

\section{A. Comparison with Conventional Cooperative Sensing}

In HASS, a CS-SU can select a favorable CS-RS according to (4), which makes the 2-hop link to have the highest equivalent SNR, denoted by HASS Scheme 1. On the other hand, a CS-SU can maintain a list of two CS-RSs by applying both (4) and (5), which correspond to reporting performance and detecting performance respectively, denoted by HASS Scheme 2. Figure 2(a) shows that HASS scheme 1 outperforms conventional cooperative sensing Scheme in [16] because hierarchical sensing with soften hard combination in HASS brings better detection performance and cooperative reporting brings better reporting performance. Further, the comparison between HASS Scheme 1 and Scheme 2 is shown in Fig. 2(b).

As an example of showing performance of these two schemes, we randomly distribute SUs in a circular cell with radius of 100 meters. Following the steps outlined in Section II.B, six SUs become CS-RSs, and the other fifteen SUs are CS-SUs. Three PUs are assumed to be randomly located in the cell, and CR-BS is at the center of the cell. The powers of PUs and SUs are assumed to be $10 \mathrm{~mW}$ and $20 \mathrm{~mW}$ respectively. Path loss exponent is set to be 3 , and the noise power is assumed to be $-100 \mathrm{dBW}$. Signal sent from CS-SUs to CS-RSs according to Step 5 in Section II.B is under Rayleigh fading. Individual $P_{d}$ is set to be 0.8 , we examine the system performance by increasing individual $P_{f}$ from 0.0005 to 0.5 .
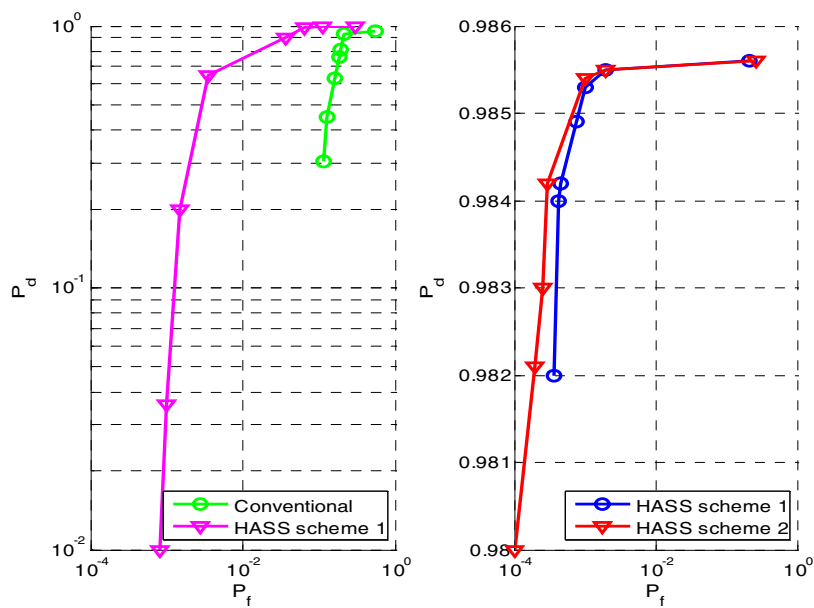

Figure 2. The comparison of spectrum sensing performance (a) HASS vs. Conventional: (b) HASS scheme1 vs. scheme2

The HASS Scheme 2 outperforms the HASS Scheme 1. The improvement could be explained as the result of the dominant effects of cooperative detection in Step 5 of the procedure explained in Section II.B. HASS scheme 2 not only bring the better reporting performance based on cooperative reporting, but also get the better detection performance due to adaptive cooperative detection. 


\section{B. Impact of AF Relay Parameters}

In HASS, the degree of amplification of the observed signal by CS-SUs before relaying to CS-RS may have substantial impacts on the system performance.

Three cases are tested here. In Case 1, all CS-SUs belonging to the same CS-RS use the same transmit power, indicating different amplification factors used by each CS-SU. In Case 2, all CS-SUs apply the same amplification factor for relaying. Case 3 is similar to Case 2, but only those CS-SUs having detected SNR above a predefined threshold can relay the observed signals. In Case 1, the transmit power is calculated by following procedures: all CS-SUs belonging to one CS-RS employ a fixed gain, and then calculate the 'virtual' transmit power, and the average power of these 'virtual' ones is used as the actual transmit power. In Case 3, the predefined threshold is the average detection SNR of those CS-SUs ready to relay the observed signals, that is, CS-SUs are categorized into two sets according to their individual detection SNRs. Those CS-SUs with higher detection SNR relay the observed signal, and the other CS-SUs simply discard their detection results. In order to enable demonstration of the effect of relay processing by having more CS-SUs falling in region 2, PU power is set to 0.15 watts, and the detection probability is set to 0.8 .

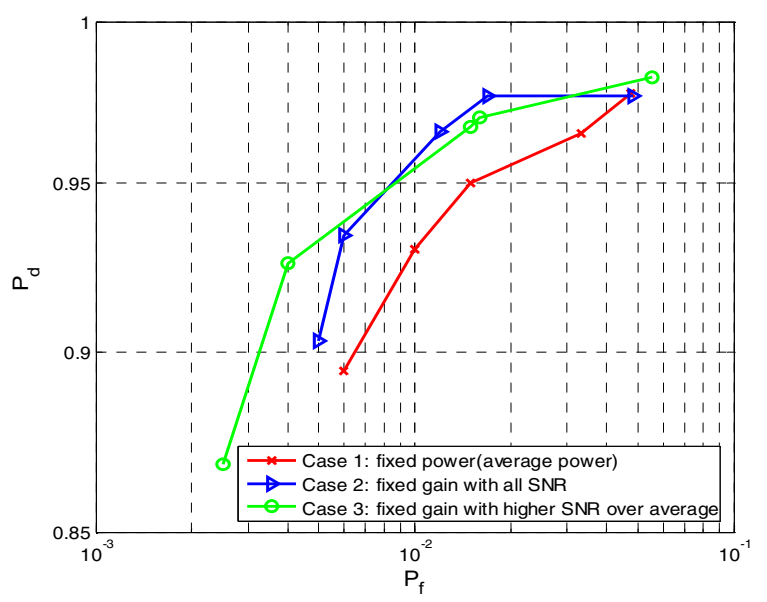

Figure 3. Impact of AF parameters

Figure 3 shows that the proposed HASS scheme has relatively poor performance for Case 1 , and the reason is that using the same transmit power for all nodes implies a greater weight for nodes with low detection SNR than that for nodes with high detection SNR. As a result, nodes with low detection SNR amplify the embedded noise to a great extent, leading to serious contamination of signals observed at CS-RS. The performances for Case 2 and Case 3 are quite similar in the high detection probability zone (higher than 0.95 on the $\mathrm{Y}$ axis). As the detection probability descends, the performance for Case 3 is better than that for Case 2, suggesting benefits brought by the use of threshold in determining whether to relay or not. In practice, the merits of use of threshold will be valuable, since it improves the detection performance while reducing the amount of information reported to CS-RSs.

\section{Impact of Data Fusion in CS-RSs}

We adopt a high $K$-to- $N$ ratio in CR-BS and a low ratio in CS-RSs in scheme 1 . On the contrast, we adopt a low $K$-to- $N$ ratio in CS-RSs and a high ratio in CR-BS in scheme 2. In the simulation, we set the value of 0.5 as the high $K$-to- $N$ ratio, and 0.3 as the low ratio. All the other parameters remain unchanged during the simulation.

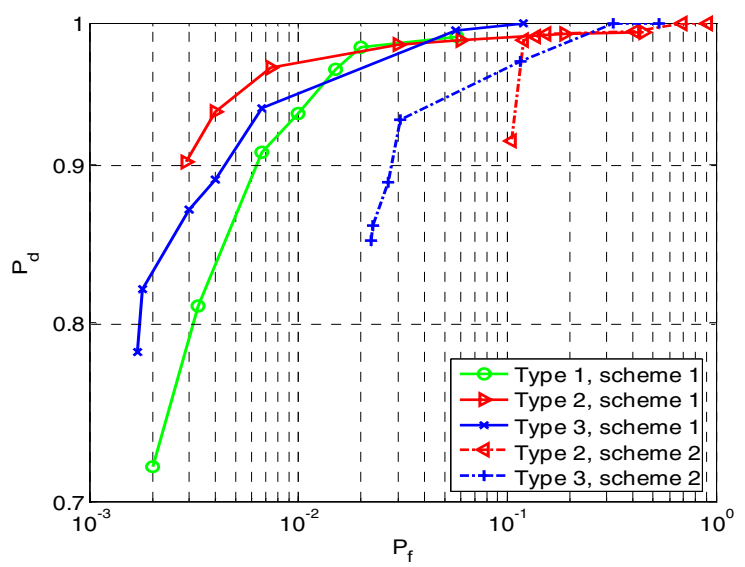

Figure 4. Impact of CS-RS fusion

Figure 4 shows the simulation results, in which each curve demonstrates the system $P_{d}-P_{f}$ relation in individual cases, as well as illustrating the dynamic range of $P_{d}$ and $P_{f} . P_{d}$ and $P_{f}$ are two detection properties of interest, since $\lambda_{1}$ in (2-1) has to be less than $\lambda_{2}$ in (2-2). It can be seen that Type 1 has the widest dynamic range of $P_{d}$ and $P_{f}$, but performs not well enough in terms of the $P_{d}-P_{f}$ relation. This is attributed to the fact that Type 1 is similar to the conventional cooperative sensing scheme where final decision is made based on oneround fusion of multiple detection results. Because of taking the OR rule, Type 2 has a relatively high detection probability, but also with a relatively wide dynamic range in terms of falsealarm probability. Type 3 has moderate performance where one detection property outperforms the other two types and another detection property underperforms them. Since a CS-RS sends local decision and relay-level decision separately to CR-BS, the degree of errors in decision fusion resulted from certain CSRSs could be reduced at CR-BS by leveraging decisions from other CS-RSs.

We can also observe the impacts of the " $K$-out-of- $N$ " rule on data fusion in Fig. 4. Solid lines in the figure are based on scheme 1 , in which the $K$-to- $N$ ratio is 0.3 for CS-RSs and 0.5 for CR-BS. Dotted lines in the figure are based on scheme 2, in which the $K$-to- $N$ ratio is 0.5 for CS-RSs and 0.3 for CR-BS. Comparing the performances of scheme 1 and scheme 2 with fusion Type 2, we find the former one significantly outperforms the latter one. A lower $K$-to- $N$ ratio at CR-BS leads to a higher false alarm probability. On the other hand, with a low $K$-to- $N$ ratio at CS-RSs, there is no significant difference in the false alarm probability for the two schemes, since the portion for fusion performed by CS-RSs is relatively 
low. We can also see that in Scheme 2 with fusion Type 2, the false-alarm probability has a higher limit about 0.12 , which is attributed to the error rate of the radio link between CS-RS and CR-BS. When $\lambda_{1}$ in (2-1) is large enough, the detecting CSSUs and CS-RSs have excellent false-alarm detection performance, thus false-alarm detection performance at CR-BS only depends on transmission error rate of the reporting link. While the bit-error-rate of transmission decreases, the average overall false-alarm rate is expected to decrease. Similar phenomena are also observed by comparing Scheme 1 and Scheme 2 with fusion Type 3 . In general, a higher $K$-to- $N$ ratio at CR-BS leads to more separate decisions from CS-RSs being taken into account by CR-BS, for making a final decision, thus errors resulted from individual CS-RSs have lower impacts on the final decision, resulting in a better performance in terms of false-alarm.

\section{CONCLUSIONS}

How to limit the interference to a primary system while making the best use of the available spectrum is arguable to the most essential spectrum sensing problem in cognitive radio networks. This paper proposes a novel Hierarchical and Adaptive Spectrum Sensing (HASS) scheme which applies hierarchical detection and combines adaptive amplify-andforward detection and decode-and-forward with soften hard detection fusion scheme.

In the proposed scheme, according to the soften hard detection fusion principle, the energy range is divided into three detection regions by two energy thresholds corresponding to the minimum detection probability and maximum false alarm probability with one-bit reporting overhead. Simulations have been conducted to investigate the impacts of relaying at cooperative sensing secondary users (CS-SUs) and data fusion at cooperative sensing relay stations (CS-RSs). From the simulation results we observe that fixed relay gain with relay threshold at CS-SUs and without combining the relay-level result and local detection result at CS-RSs can obtain better detection performance. Moreover, simulation results also show that the sensing performance of the proposed scheme is improved considerably in comparison to the conventional cooperative sensing scheme.

\section{ACKNOWLEDGMENT}

This work was supported in part by the National High Technology Development 863 Program of China under Grant 2009AA011801.

\section{REFERENCES}

[1] X. J. Li, B.-C. Seet, and P. H. J. Chong, "Multihop cellular networks: Technology and economics," Elsevier's Computer Networks, vol. 52, no. 9, pp. 1825-1837, June 2008.

[2] I. F. Akyildiz, W.-Y. Lee, M.C. Vuran, and S. Mohanty, "NeXt Generation/dynamic spectrum access/cognitive radio wireless networks: A survey," Elsevier's Computer Networks, vol. 50, no.13, pp. 2127 2159, September 2006.
[3] A. Ghasemi and E. S. Sousa, "Opportunistic spectrum access in fading channels through collaborative sensing," Journal of Communications (JCM), 2(2), pp. 71-82, March 2007.

[4] X. Jing and D. Raychaudhari, "A spectrum etiquette protocol for efficient coordination of radio devices in unlicensed bands," in Proc. Personal, Indoor, Mobile Radio Communications Conf. (PIMRC), Beijing, 2003.

[5] H. Urkowitz, "Energy detection of unknown deterministic signals," Proceedings of the IEEE, 55 (4), pp. 523-531, 1967.

[6] F. F. Digham, M. S. Alouini, and M. K. Simon, "On the energy detection of unknown signals over fading channels," in Proc. IEEE Int. Conf. on Commun. (ICC), vol. 5, pp. 3575-3579, May 2003.

[7] J. Hillenbrand, T. A. Weiss, and F. K. Jondral, "Calculation of detection and false alarm probabilities in spectrum pooling systems," IEEE Commun. Letters, vol. 9, no. 4, pp. 349-351, April 2005.

[8] D. Cabric, S. M. Mishra, and R. W. Brodersen, "Implementation issues in spectrum sensing for cognitive radios," in Proc. 38th Asilomar Conference on Signals, Systems and Computers, pp. 772-776, November 2004.

[9] S. Enserik and D. Cochran, "A cyclostationary feature detector," in Proc. 28th Asilomar Conference on Signals, Systems and Computers, Pacific Grove, CA, October 1994.

[10] B. Wild and K. Ramchandran, "Detecting primary receivers for cognitive radio applications," in Proc. IEEE Int. Symp. on New Frontiers in Dynamic Spectrum Access Networks (DySPAN), pp. 124130, November 2005.

[11] S. M. Mishra, A. Sahai, and R.W. Broderson, "Cooperative sensing among cognitive radios," in Proc. IEEE Int. Conf. on Commun. (ICC), Istanbul, Turkey, June 2006.

[12] A. Ghasemi and E. S. Sousa, "Collaborative spectrum sensing for opportunistic access in fading environments", in Proc. IEEE Int. Symp. on New Frontiers in Dynamic Spectrum Access Networks (DySPAN), pp. 131-136, November 2005.

[13] J. Ma and Y. (G.) Li, "Soft combination and detection for cooperative spectrum sensing in cognitive radio networks," in Proc. IEEE Globecom, Washington, D. C., November 2007.

[14] T. C. Aysal, A. R. Biswas, K. Sitham, and R. Piesiewicz, "Cooperative spectrum sensing and cognitive broadcast signaling," in Proc. World Wireless Research Forum (WWRF) \#21, Stockholm, Sweden, 2008.

[15] P. Qihang, Z. Kun, W. Jun, et al. "A distributed spectrum sensing scheme based on credibility and evidence theory in cognitive radio context," in Personal, Indoor, Mobile Radio Communications Conf. (PIMRC), Helsinki, September 2006.

[16] L. Yi, Research on the Cooperative Communication Algorithms in Future Wireless Networks, Ph.D. dissertation, Beijing University Posts and Telecommunications (BUPT), 2008.

[17] P. Pawełczak, C. Guo, R. Venkatesha Prasad, and R. Hekmat, "Clusterbased spectrum sensing architecture for opportunistic spectrum access networks," IRCTR-S-004-07 Report 12, February 2007.

[18] C. Sun, W. Zhang, and K. B. Letaief, "Cluster-based cooperative spectrum sensing for cognitive radio systems," in Proc. IEEE Int. Conf. on Commun. (ICC 2007), pp. 2511-2515, Glasgow, Scotland, UK, June 2007.

[19] A. Wald, Sequential Analysis, New York: Wiley, 1947.

[20] H. Li, H. Yu, H. W. Luo, J. Guo, and C. Li, "Dynamic subchannel and power allocation in OFDMA-based DF cooperative relay networks," in Proc. IEEE Globecom, November/December 2008.

[21] R. V. Hogg and A. T. Craig, Introduction to Mathematical Statistics, $4^{\text {th }}$ edition. New York: Macmillan, 1978.

[22] X.-W. Zhou, J. Ma, G. Y. Li, Y. H. Kwon, and A. C. K. Soong, "Probability-based combination for cooperative spectrum sensing in cognitive radio networks," in Proc. IEEE 2009 Int. Conf. on Commun. (ICC), Dresden, Germany, June 2009. 